\title{
Cognitive and Physical Assessment in the Elderly while Maintaining Social Distance Using A Web Conference System: A Pilot Study
}

Noboru Hasegawa $^{{ }^{*}}$, Takako Yamada ${ }^{2}$, Miyako Mochizuki ${ }^{3}$, Yoshihito Tsubouchi ${ }^{4}$, Nobuyuki Honda ${ }^{5}$ and Nobuko Shimizu ${ }^{6}$

${ }^{1}$ Graduate School of Nursing, Doshisha Women's College, Kodo, Kyotanabe, Kyoto 610-0395, Japan

${ }^{2}$ Bukkyo University, 7, Higashitoganoo-cho, Nishinokyo, Nakagyo-ku, Kyoto604-8418, Japan

${ }^{3}$ Kyoto Bunkyo Junior College, 80 Senzoku, Makishima-cho, Uji, Kyoto 611-0041, Japan

${ }^{4}$ Naragakuen University, 3-15-1 Nakatomigaoka, Nara 631-8524, Japan

${ }^{5}$ Kansai University of Welfare Sciences, 3-11-1 Asahigaoka, Kashiwara, Osaka 582-0026, Japan

${ }^{6}$ Toyama Prefectural University, 2-2-78 Nishinagae, Toyama 930-0975, Japan

\section{Abstract}

Background: We studied whether cognitive and physical assessments of the elderly can be carried out as reliably via a web conference system with social distancing as they can face-to-face.

Methods: We enrolled 10 male and 1 female elderly Japanese aged 69-80 years. The application of an audiovisual technology setup using the Zoom web conference system enabled assessment with the examinee and examiner in separate rooms. For assessment of cognitive function, 2 personal computers (PCs), one with a webcam and one with a document camera, were located in the examinee room (one shows the examinee's face and the other shows the drawing the examinee made on the desk). The examiner's PC with webcam was connected to the web conference system (Zoom as a host). For assessment of physical function, one PC with webcam was located in the examinee room to show the examinee's movement during the physical function test (5-m walking speed), and the examiner assessed physical strength (upper grip, chair standing 30 seconds (CS-30)) via another PC through a video camera-image connected by video-capture board. The examiner's PC with webcam was connected to the web conference system (Zoom as a host) for oral advice. An action camera was attached to the waist of the examinee and 5-m walking speed was assessed from pictures of the floor and the examinee's lower limbs after walking.

Results: The relationship between the Mini Mental State Examination (MMSE) in a face-to-face setting and the Montreal Cognitive Assessment, Japanese version (MoCA-J) via web conference was similar to the relationship between the MMSE via web conference and MoCA-J face-to-face. The physical assessment (5-m walking test at maximum speed, upper grip, CS-30) via web conference was similar the results with face-to-face assessment.

Conclusion: These results suggest that a web conference system for measurement of cognitive and physical function is equally beneficial to face-to-face assessment.

\section{Introduction}

The emergence of COVID-19 has resulted in disruption of the daily routine and habits of billions of people across the globe. Many people have been left in their homes and many of these changes have created additional barriers for the elderly in maintaining cognitive and physical activity. It is necessary to measure home-based physical activity and cognitive function in the elderly while maintaining social distancing.

In the area of health, internet-based intervention for depression [1], remote clinical conference (teleneuropsychology) [2], teleconsultation [3], and online rehabilitation for chronic obstructive pulmonary disease [4] have been conducted. An online video conferencing system showed the same Mini Mental State Examination (MMSE) score as inperson tests in subjects without cognitive impairment (27.6) [5]. The Montreal Cognitive Assessment (MoCA) was conducted in person and remotely via web-based conference by email, printed matter and webcam in individuals with movement disorders [6]. With webbased tests, however, the examinees had to hold up their drawings to the camera for real time scoring. This is difficult for examinees with cognitive impairment or low MMSE score. An online video-facilitated exercised program (6 min walk distance, 6 min walk test) with no specific advice was less effective than face-to-face exercise with direct discussion [3].

\section{Publication History:}

Received: April 15, 2021 Accepted: May 04, 2021

Published: May 06, 2021

\section{Keywords:}

Cognitive assessment, Physical assessment, Social distancing, Web conference system
Therefore, the present study was designed to investigate the efficacy and safety of online assessments using the Zoom web conference system (e.g. microphone, speaker, document camera, webcam, GoPro, video camera) compared with traditional face-to-face assessment of cognitive and physical function while maintaining social distancing.

\section{Methods}

\section{Subjects and setting}

Prior to the study, approval was obtained from the ethics committee of Kyoto Bunkyo Junior College (project registration number in 2016: 7). A total of 11 healthy adults age $\geq 69$ years were included.

"Corresponding Author: Prof. Noboru Hasegawa, Graduate School of Nursing, Doshisha Women's College, Kodo, Kyotanabe, Kyoto 610-0395, Japan, Tel: +81774-65-8855, Fax: +81-774-65-8820; E-mail: nhasegaw@dwc.doshisha.ac.jp

Citation: Hasegawa N, Yamada T, Mochizuki M, Tsubouchi M, Honda N, et al. (2021) Cognitive and Physical Assessment in the Elderly while Maintaining Socia Distance Using A Web Conference System: A Pilot Study. Int J Nurs Clin Pract 8: 340. doi: https://doi.org/10.15344/2394-4978/2021/340

Copyright: (c) 2021 Hasegawa et al. This is an open-access article distributed under the terms of the Creative Commons Attribution License, which permits unrestricted use, distribution, and reproduction in any medium, provided the original author and source are credited. 
Citation: Hasegawa N, Yamada T, Mochizuki M, Tsubouchi M, Honda N, et al. (2021) Cognitive and Physical Assessment in the Elderly while Maintaining Social Distance Using A Web Conference System: A Pilot Study. Int J Nurs Clin Pract 8: 340. doi: https://doi.org/10.15344/2394-4978/2021/340

Page 2 of 5

\section{Cognitive function test}

The application of the audiovisual technology setup using the Zoom web conference system enabled assessment with the examinee and examiner in separate rooms (Figure 1). Two personal computers (PCs), one with a webcam and one with a document camera, were located in the examinee room (one shows the examinee's face image and the other shows the drawings the examinee made on the desk with no adaptation in administrating the visual subsection). The examiner's PC with webcam was connected to the web conference system (Zoom as a host) and could exchange information with the 2 examinee PCs. The examinee could see the examiner's face through a front PC screen, and the examiner asked a question while looking at the examinee's face and drawn image. An assistant was available as needed. The MMSE and MoCa-J were used to assessment cognitive function. All participants were randomly assigned two groups. In one, the assessment was made with MMSE via the Internet and face-toface MoCa-J, while in the other group this was reversed. The same examiner performed the 2 measurements (face-to-face and Internet) for the same subject.

The MMSE was used as a cognitive function test. It consists of five downstream items of orientation, memory, attentiveness for calculations, speech function, and design capacity. The maximum score for the MMSE was 30 points, and the cutoff score for dementia was 23 to 24 points [7]. The MoCA-J may be better at detecting early cognitive dysfunction and was also used as a cognitive function test. The maximum score for the MoCA-J was 30 points, and cutoff score for dementia was 25 to 26 points [8]. These tests were performed by verbal questioning for $5 \mathrm{~min}$ to $10 \mathrm{~min}$ by skilled occupational therapists.

\section{Physical function test}

The application of the audiovisual technology setup using the Zoom web conference system enabled assessment with the examinee and examiner in separate rooms (Figure 2). One PC with a webcam was located in the examinee room to show the examinee's movement during the physical function test ( $5 \mathrm{~m}$ walking speed), and the examiner assessed physical strength (upper grip, CS-30) via another PC through a video camera-image connected by video-capture board.

The examiner's PC with webcam was connected to the web conference system (Zoom as a host) and the examinee saw the examiner's face through the PC screen. The examiner gave advice while watching the examinee's movement. The examinees were asked to walk down an $11 \mathrm{~m}$ walk-way at their preferred and maximum walking speeds. An action camera (HERO7, GoPro, Inc., CA, USA) was attached to the waist of the examinee and $5 \mathrm{~m}$ walking speed was assessed between meters $3 \mathrm{~m}$ and $8 \mathrm{~m}$ from the picture of the floor and the examinee's lower limbs after walking. An assistant was available as needed. The test was performed by skilled occupational therapists.

\section{Statistical analysis}

The differences in cognitive and physical function between the different groups (web conference vs. face-to-face group) were evaluated using Pearson's correlation coefficients. A p-value of $<0.05$ was considered to be statistically significant. Analyses were carried out using SPSS 21 for Windows (IBM, Japan).

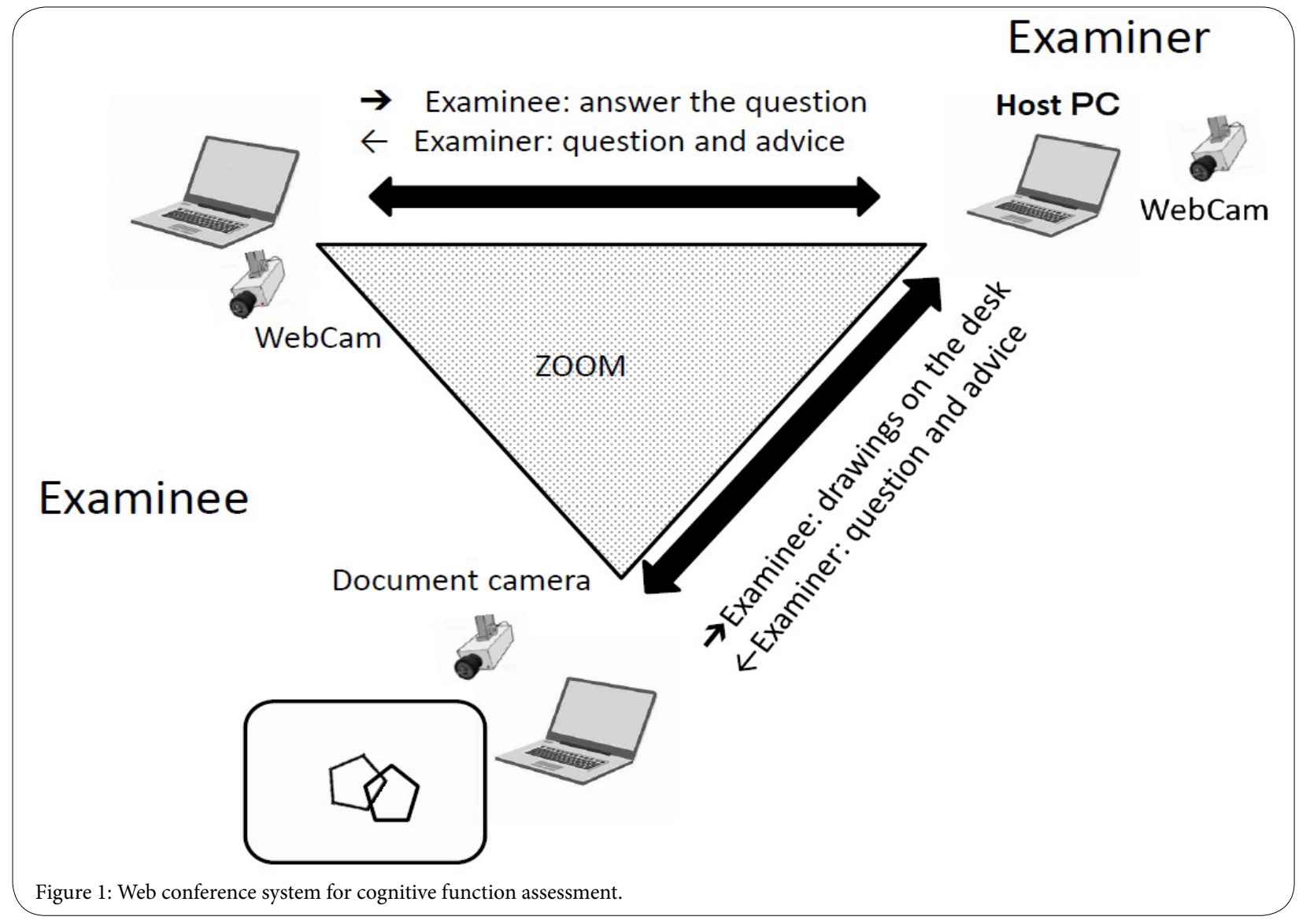


Citation: Hasegawa N, Yamada T, Mochizuki M, Tsubouchi M, Honda N, et al. (2021) Cognitive and Physical Assessment in the Elderly while Maintaining Social Distance Using A Web Conference System: A Pilot Study. Int J Nurs Clin Pract 8: 340. doi: https://doi.org/10.15344/2394-4978/2021/340

Page 3 of 5

\section{Results and Discussion}

\section{Study subjects}

The characteristics of the study subjects are shown in Table 1. Mean age was 74.2 years for males $(n=10)$ and 80.0 years for females $(\mathrm{n}=1)$. Obesity was defined as a body-mass index (BMI) $\geq 25 \mathrm{~kg} / \mathrm{m}^{2}$. The prevalence of obesity defined from BMI was $30.0 \%$ in males.

\section{Cognitive function}

The relationships between MMSE and MoCA-J scores are shown in Figure 3. The relationship between face-to-face MMSE and

\begin{tabular}{|l|l|}
\hline Age & $74.7 \pm 3.3$ \\
\hline Body height & $167.0 \pm 6.9$ \\
\hline Body weight & $66.0 \pm 10.3$ \\
\hline BMI & $23.6 \pm 2.3$ \\
\hline
\end{tabular}

MoCA-J via web conference showed a strong correlation $(\gamma=0.860)$. The relationship between MMSE via web conference and face-to-face MoCA-J showed a slightly strong correlation $(r=0.587)$. The values on the X-axis were the same as the cutoff score for dementia (MoCA-J 23, MMSE 25) in both two groups. These results suggest that there is not
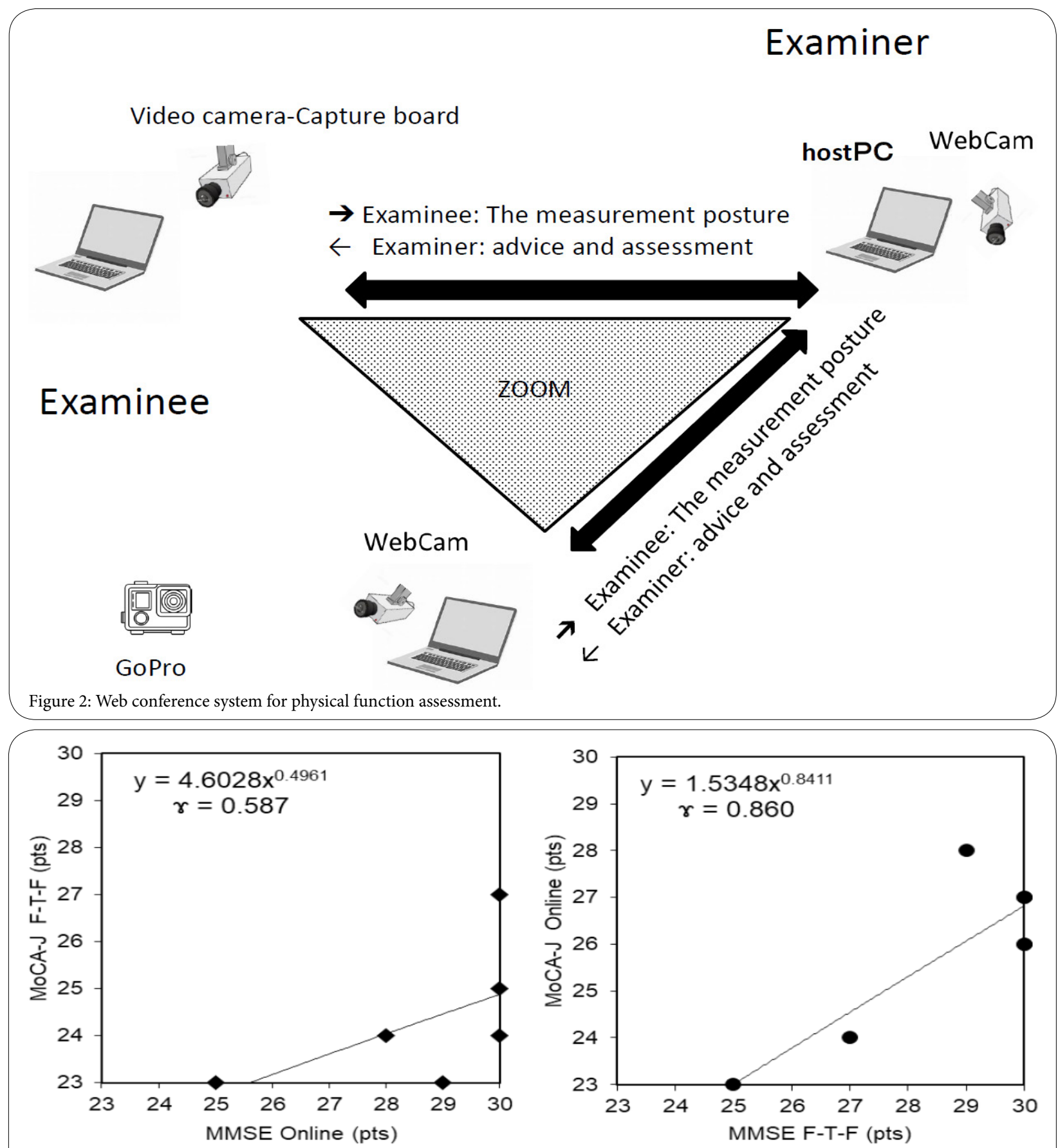

Figure 3: Cognitive function assessment between-group differences. 

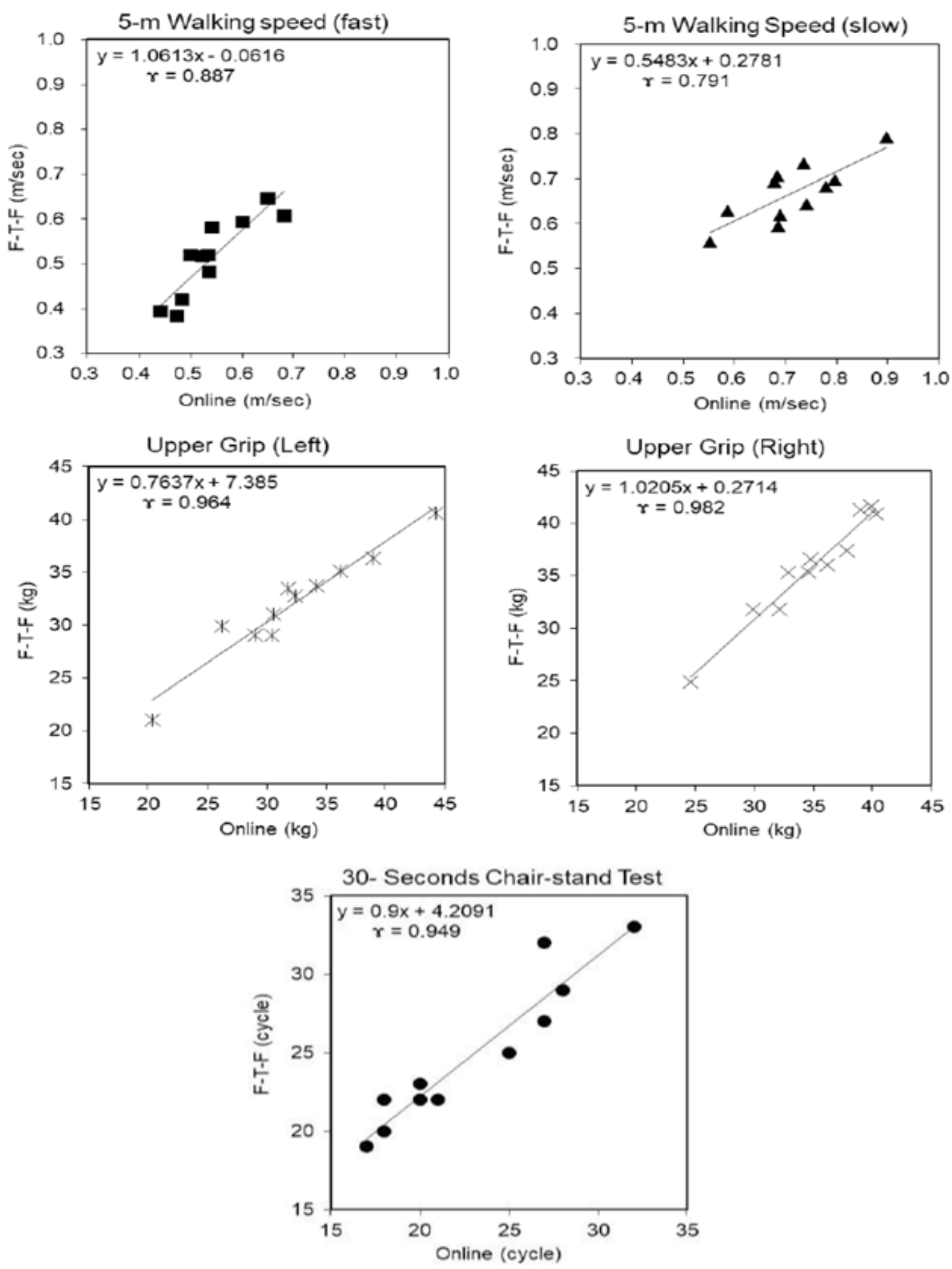

Figure 4: Physical function between-group differences. 
Citation: Hasegawa N, Yamada T, Mochizuki M, Tsubouchi M, Honda N, et al. (2021) Cognitive and Physical Assessment in the Elderly while Maintaining Social Distance Using A Web Conference System: A Pilot Study. Int J Nurs Clin Pract 8: 340. doi: https://doi.org/10.15344/2394-4978/2021/340

Page 5 of 5

a significant difference between the two groups (web conference vs. face-to-face group) and that the web conference system is effective for the health management of elderly people.

\section{Physical function}

The physical assessments ( $5 \mathrm{~m}$ walking speed, upper grip and CS30 ) via web conference and face-to-face assessment had a significant strong positive correlation (Figure $4, p<0.05$ ). These measurement values show generally good agreement except for $5 \mathrm{~m}$ walking speed at examinee's preferred walking speed. When examinees were by themselves (online), the walking speed tended to be faster than in the presence of an examiner (face-to-face).

These results revealed that the result of $5 \mathrm{~m}$ walking speed at examinee's preferred walking speed was unusable.

These findings show that it is not necessary for examinees and skilled occupational or physical therapists to be present at the same location for cognitive and physical assessments to be carried out. In video consultations for people living with asthma, participants found very little difference between an online and a face-to-face consultation approach [9]. The intraclass correlation coefficients were high for the MoCA-J total score between video teleconferencing and face-to-face conferences. These results revealed that assessment from the home is possible if the web environment is set up for it. However, there are many limitations to this study. It is not clear how the level of cognitive impairment might impact the validity of web conferences. Even if the audio and video transmission system has no problems, it is unknown whether the examinee completely understands and complies with the examiner's instruction.

This is a preliminary study with very small numbers and was uncontrolled. Further study with larger numbers is warranted.

\section{Conclusion}

These results suggest that a web conference system for cognitive and physical function assessment is equally beneficial to face-to-face assessment.

\section{Competing Interest}

The authors declare that they have no competing interests.

\section{Author Contributions}

Dr. Hasegawa was responsible for the study conception, design, analysis, interpretation of data, and drafting of the manuscript.

Ms. Mochizuki was responsible for data acquisition and proof-reading of the manuscript, and she participated in the data analysis.

Dr. Yamada was responsible for the data acquisition and proofreading of the manuscript.

Ms. Tsubouchi was responsible for the data acquisition and proofreading of the manuscript.

Ms. Honda was responsible for the data acquisition and proof-reading of the manuscript.

Dr. Shimizu was responsible for the data acquisition and proofreading of the manuscript.

\section{Acknowledgements}

Dr. Masashi Kume helped in trials of the various web conferencing hardware. The authors acknowledge the technical assistance of the PC support desk in the Kyoto Bunkyo University General Administration Division

\section{Funding}

This work was supported by KAKENHI (grant numbers 17K12534 and 18K10611).

\section{References}

1. Wagner $B$, Horn AB, Maercker A (2014) Internet-based versus face-to-face cognitive-behavioral intervention for depression: a randomized controlled non-inferiority trial. J Affect Disord 154: 113-121.

2. Tailby C, Collins AJ, Vaughan DN, Abbott DF, O'Shea M, et al. (2020) Teleneuropsychology in the time of COVID-19: The experience of The Australian Epilepsy Project. Seizure 83: 89-97.

3. Kirkwood KT, Peck DF, Bennie L (2006) The consistency of neuropsychological assessments performed via telecommunication and face to face. J Telemed Telecare 6: 147-151.

4. Bourne S, DeVos R, North M, Chauhan A, Green B, et al. (2017) Online versus face-to-face pulmonary rehabilitation for patients with chronic obstructive pulmonary disease: randomised controlled trial. BMJ Open 17: e014580.

5. Cullum CM, Hynan LS, Grosch M, Parikh M, Weiner MF, et al. (2014) Teleneuropsychology: evidence for video teleconference-based neuropsychological assessment. J Int Neuropsychol Soc 20: 1028-1033.

6. Abdolahi A, Bull MT, Darwin KC, Venkataraman V, Grana MJ, et al. (2016) A feasibility study of conducting the Montreal Cognitive Assessment remotely in individuals with movement disorders. Health Informatics J 22: 304-311.

7. Folstein MF, Folstein SE, McHugh PR (1975) "Mini-mental state". A practical method for grading the cognitive state of patients for the clinician. J Psychiatr Res 12: 189-198.

8. Nasredding ZS, Phillips N, Bédirian V, Charbonneau S, Whitehead V, et al. (2005) The Montreal Cognitive Assessment, MoCA: a brief screening Tool for Mild Cognitive Impairment. J Am Geriatr Soc 53: 695-699.

9. Hamour O, Smyth E,Pinnock H (2020) Completing asthma action plans by screen-sharing in video-consultations: practical insights from a feasibility assessment. NPJ Prim Care Respir Med 30: 46-48.

10. Iiboshii K, Yoshida K, Yamaoka Y, Eguchi Y, Sato D, et al. (2020) A validation study of the remotely administered Montreal Cognitive Assessment tool in the elderly Japanese Population. Telemedicine and e-Health 26: 920-928. 